\title{
Density-Based Spatial Outliers Detecting
}

\author{
Tianqiang Huang ${ }^{1}$, Xiaolin Qin ${ }^{1}$, Chongcheng Chen $^{2}$, and Qinmin Wang ${ }^{2}$ \\ ${ }^{1}$ Department of Computer Science and Engineering, \\ Nanjing University of Aeronautics and Astronautics, Nanjing, 210016, China \\ tianqianghuang@163.com \\ ${ }^{2}$ Spatial Information Research Center in Fujian Province, \\ Fuzhou, 350002, China \\ http://www.sirc.gov.cn/
}

\begin{abstract}
Existing work in outlier detection emphasizes the deviation of non-spatial attribution not only in statistical database but also in spatial database. However, both spatial and non-spatial attributes must be synthetically considered in many applications. The definition synthetically considered both was presented in this paper. New Density-based spatial outliers detecting with stochastically searching approach (SODSS) was proposed. This method makes the best of information of neighborhood queries that have been detected to reduce many neighborhood queries, which makes it perform excellently, and it keeps some advantages of density-based methods. Theoretical comparison indicates our approach is better than famous algorithms based on neighborhood query. Experimental results show that our approach can effectively identify outliers and it is faster than the algorithms based on neighborhood query by several times.
\end{abstract}

\section{Introduction}

A well-quoted definition of outliers is the Hawkin-Outlier [1]. This definition states that an outlier is an observation that deviates so much from other observations as to arouse suspicion that it was generated by a different mechanism. However, the notion of what is an outlier varies among users, problem domains and even datasets[2]: (i) different users may have different ideas of what constitutes an outlier, (ii) the same user may want to view a dataset from different "viewpoints" and, (iii) different datasets do not conform to specific, hard "rules" (if any).

We focus on outlier in spatial database, in which objects have spatial and non-spatial attributions. Such datasets are prevalent in several applications. Existing work of Multidimensional outlier detection methods can be grouped into two sub-categories, namely homogeneous multidimensional and bipartite multi- dimensional methods [3]. The homogeneous multidimensional methods model data sets as a collection of points in a multidimensional isometric space and provide tests based on concepts such as distance, density, and convex hull depth. These methods do not distinguish between spatial dimensions and attribute dimensions (non-spatial dimensions), and use all dimensions for defining neighborhood as well as for comparison. Another multidimensional outlier detection method is bipartite multidimensional test which is designed to detect spatial outliers. They differentiate between spatial and non-spatial 
attributes. However, they defined outlier as "spatial outlier is spatially referenced objects whose non-spatial attribute values are significantly different from those of other spatially referenced objects in their spatial neighbor- hoods $[3,4]$ ", which emphasizes non-spatial deviation and ignores spatial deviation.

In some application, domain specialist needs detect the spatial objects, which have some non- spatial attributes, deviation from other in spatial dimension. For example, in image processing, detecting a certain type vegetable is anomaly in spatial distribution. The vegetable type is non-spatial attribute, and the vegetable location means spatial attributes. As another example, government wants to know middle incoming residents distribution in geo-space. To detect outliers in these instances, spatial and non-spatial attributes may be synthetically taken into account. For example, there are two type objects in Fig. 1. The solid points and rings respectively represent two objects with different non-spatial attribute, such as the solid objects represent one vegetable and the rings are the other. All objects in Fig. 1 are one cluster when we didn't consider non-spatial attribute, but they would have different result when we took spatial and non-spatial attribute into account. Apparently, when we focus solid objects, the solid objects in $C 1$ and $C 2$ are clusters, and object $a$ and $b$ are outliers.

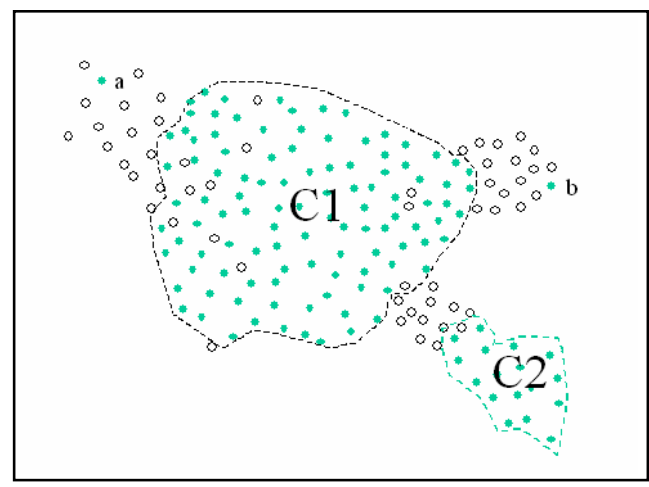

Fig. 1. An illumination example

We took into account of spatial and non-spatial attributes synthetically to define the outliers. If the objects that have some non-spatial attributes are keep away from their neighbor in spatial relation. We defined them outliers.

The main contributions of this paper are: (1) we propose a novel density-based algorithm to detect it, which is the quicker than existing algorithms based on neighborhood query. (2) We evaluate it on both theory and experiments, which demonstrate that algorithm can detect outlier successfully with better efficiency than other algorithms based on neighborhood query.

The remainder of the paper is organized as follows: In section 2, we discuss formal definition of outliers. Section 3 presents the SODSS algorithm. Section 4 evaluates performance of SODSS. Section 5 reports the experimental evaluation. Finally, Section 6 concludes the paper. 


\section{Density-Based Notion of Spatial Outliers}

In this section we present the new definition of outlier, in which spatial and non-spatial attributes were synthetically taken into account.

Given a dataset $D$, a symmetric distance function dist, parameters Eps and MinPts, and variable attrs indicates the non-spatial attributes.

Definition 1. The impact neighborhood of a point $\mathrm{p}$, denoted by $I N_{E p s}(p)$, is defined as $I N_{E p s}(p)=\{q \in D \mid \operatorname{dist}(p, q) \leq$ Eps and q.attrs satisfy $\mathrm{C}\}$.

Definition 2. The Neighbor of $p$ is any point in impact neighborhood of $p$ except $p$.

Definition 3. If a point's impact neighborhood has at least MinPts points, the impact neighborhood is dense, and the point is core point.

Definition 4. If a point's impact neighborhood has less than MinPts points, the impact neighborhood is not dense. If a point is a neighbor of core point, but his neighborhood is not dense, the point is border point.

Definition 5. If a point is core point or border point, and it near a border point $\mathrm{p}$, the point is near-border point of $p$.

Definition 6. A point $p$ and a point $q$ are directly density-reachable from each other if (1) $p \in I N_{E p s}(q),\left|I N_{E p s}(q)\right| \geq \operatorname{MinPts}$ or (2) $q \in I N_{E p s}(p),\left|I N_{E p s}(p)\right| \geq \operatorname{MinPts}$.

Definition 7. A point $p$ and a point $q$ are density-reachable from each other, denoted by $\operatorname{DR}(p, q)$, if there is a chain of points $p_{1}, \ldots, p_{\mathrm{n}}, p_{1}=q, p_{\mathrm{n}}=p$ such that $p_{\mathrm{i}+1}$ is directly density-reachable from $p_{\mathrm{i}}$ for $1 \leq i \leq n-1$.

Definition 8. A cluster $C$ is a non-empty subset of $D$ satisfying the following condition: $p, q \in D$ : if $p \in C$ and $D R(p, q)$ holds, then $q \in C$.

Definition 9. Outlier $p$ is not core object or border object, i.e., $p$ satisfying the following conditions: $P \in D,|I N(p)|<$ MinPts, and $\forall q \in D$, if $|I N(q)|>$ MinPts, then $p \notin I N(q)$.

\section{SODSS Algorithm}

In $D B S C A N$ [5] or GDBSCAN [6], to guarantee finding density-based clusters or outliers, determining the directly density-reachable relation for each point by examining the neighborhoods is necessary. However, performing all the region queries to find these neighborhoods is very expensive. Instead, we want to avoid finding the neighborhood of a point wherever possible. In our method, the algorithm discards these dense neighborhoods in first, because these objects in it are impossibly outliers. The algorithm stochastically researched in database but not scan database one by one to find the neighborhood of every point like $D B S C A N$, so the algorithm outperform famous algorithms based on neighborhood query, such as DBSCAN [5], GDBSCAN [6], $L O F[7]$.

In the following, we present the density-based Spatial Outlier Detecting with Stochastically Searching (SODSS) algorithm. SODSS is consisted of three segments. The first (lines 3 17) is Dividing Segment, which divide all object into three parts, cluster set, candidate set or outlier; The second (lines 19 23) is Near-border Detecting 
Segment, which detect and record the near-border objects of candidate, i.e., the neighbors of these border objects that may be labeled candidate, which would be used to detect these border objects in the third segment; The third (lines 24 31) is Fining Segment, using the near-border objects to find these border objects and remove them.

SODSS starts with an arbitrary point $p$ and Examine its impact neighborhood NeighborhoodSet with D.Neighbors(p, Eps) in line 5. If the size of NeighborhoodSet is at least MinPts, then $p$ is a core point and its neighbors are belong to some clustering, to put them into clustering set list; otherwise, if the size is $0, p$ is outlier, so put them into outlier set; or else $p$ and his neighbor may be outliers, so put them into candidate set. Lines 19 23 detect neighbors of these that were labeled candidates in Dividing Segment and include them into candidate set. These objects would be used to detect border objects that are not outliers from candidate set. Lines 24 31 check every object in candidate set to remove the border objects.

\section{SODSS algorithm}

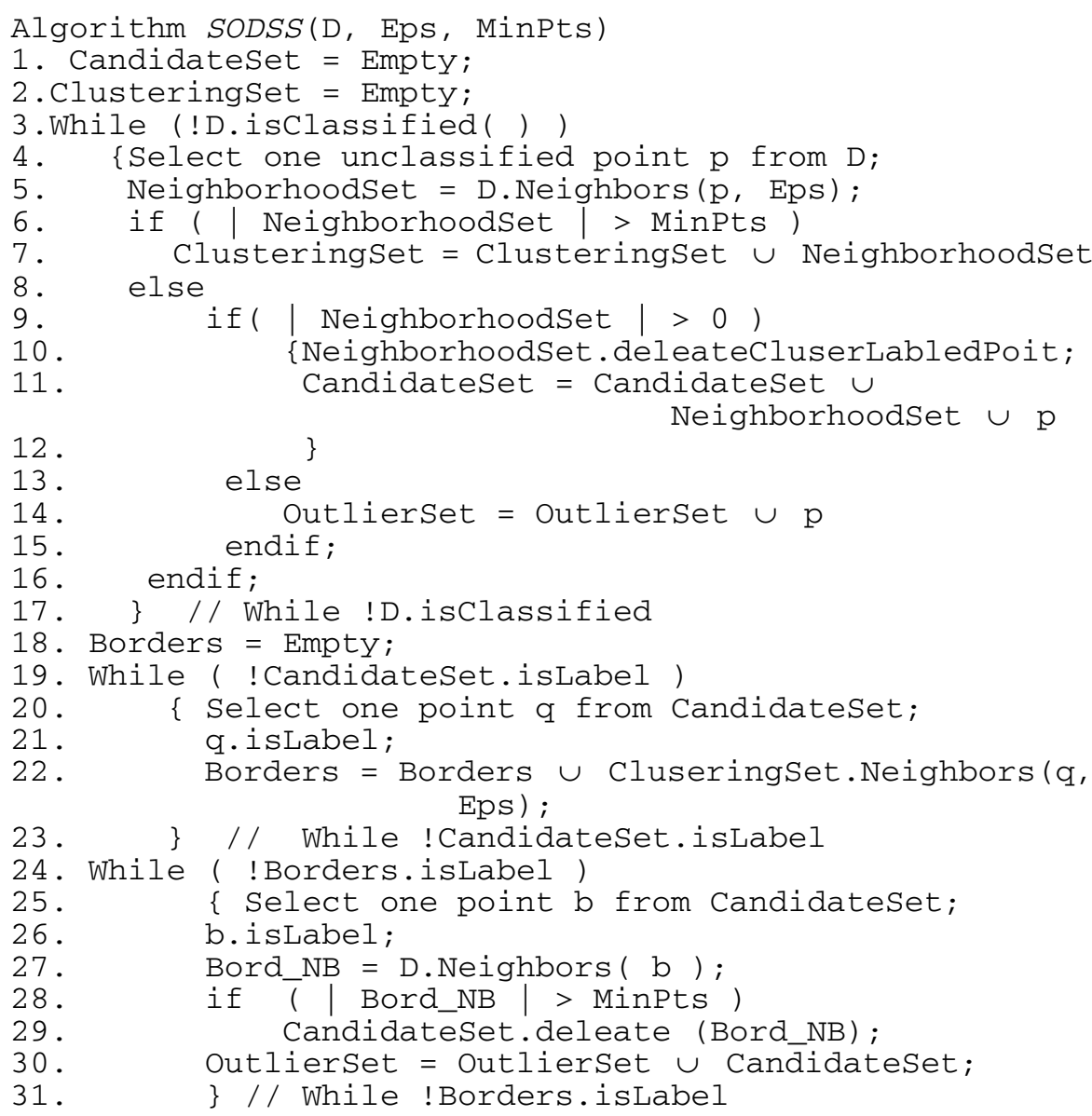


To understand this algorithm, we give example as Fig. 2. There are two type objects in Fig. 2. The solid point represented one-type objects and the ring represented the other type objects. Supposing we focus on solid objects. Apparently, there are two clusters and two outliers in solid objects in the figure. Clusters are located in center and right down, and outliers are object $a$ and object $d$. when algorithm run lines 3 17 to divide spatial objects to three parts, cluster set, outlier or candidate set. Algorithm may select object $a$, and calculate neighborhood $A$. Supposing object $b$ and $c$ have not been labeled in any dense neighborhood. They are the neighbors in neighborhood $A$, and neighborhood $A$ is sparse, so they are labeled to candidate. When object $b$ and $c$ is included in candidate set, the near-border objects near $b$ and $c$, which include in the red polygon $P$ in Fig. 2., are also included in candidate set through the Near-border Detecting Segment in line 19 23. Some of near-border objects in red polygon $P$ are dense, so object $b$ and $c$ would be removed from candidate set. So SODSS can identify real outlier.

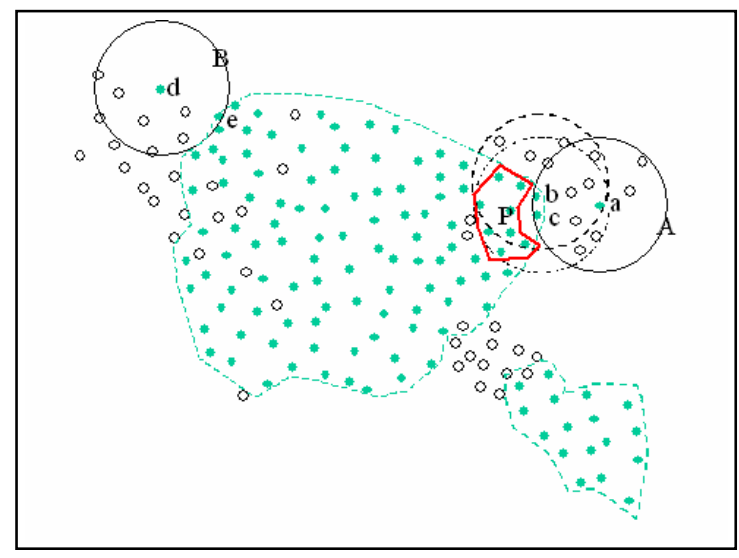

Fig. 2. Object $a$ and $d$ are outliers. Object $b$ and $c$ would be labeled candidates. The objects in red polygon $P$ are border objects that are put into candidate set in Near-border Detecting Segment

\section{Theoretical Performance Comparison of SODSS and the Other Density-Based Algorithm}

There are many density-based algorithm that were proposed to detect outliers, but calculation efficiency is not obviously improved. In the worst case, the time cost of the algorithms are $\mathrm{O}\left(n^{2}\right)$. SODSS outperform existing algorithms in calculation efficiency.

The neighborhood query D.Neighbors(p,Eps) in line 5 is the most time-consuming part of the algorithm. A neighborhood query can be answered in $\mathrm{O}(\log n)$ time using spatial access methods, such as $\mathrm{R}^{*}$-trees [8] or SR-trees [9]. When any clusters are found, their neighbors would not be examine by SODSS again, so SODSS will perform fewer neighborhood queries and save much time. Clustering objects must much more than outlier objects, so SODSS can reduce much neighborhood query and then have 
good efficiency. Supposing SODSS performs $k$ neighborhood queries, its time complexity is $\mathrm{O}(k \log n)$, which $k$ is much smaller than $n$. In the second and third segment algorithm must query neighborhood again, but these operation are in candidate set and the number of candidate is very few. The $k$ is related to Eps, so the time complexity is related to Eps. With increasing of Eps time cost decreases in certain range, however, the candidates would increase greatly when Eps exceeds the threshold and the time cost would increase obviously.

\subsection{Performance Comparison of SODSS and GDBSCAN}

GDBSCAN [6] extended the famous algorithm DBSCAN to apply to spatial database. GDBSCAN identify spatial outlier through detecting cluster, i.e., the noises are outliers. This algorithm scans database and examine all objects neighborhoods.

Eps-Neighborhood of GDBSCAN corresponds to impact neighborhood of SODSS, which is expensive operation. One crucial difference between GDBSCAN and SODSS is that once SODSS has labeled the neighbors as part of a cluster, it does not examine the neighborhood for each of these neighbors. This difference can lead to significant time saving, especially for dense clusters, where the majority of the points are neighbors of many other points.

\subsection{Performance Comparison of SODSS and $\mathrm{LOF}$}

$L O F$ [7] calculates the outlier factor for every object to detect outliers. It is the average of the ratio of the local reachability density of $p$ and those of $p$ 's MinPts-nearest neighbors. The local reachability density is based on MinPts-nearest neighbors. LOF must calculate $k$-distance neighborhoods of all objects, which time costs are equal to impact neighborhoods query. Calculating $k$-distance neighborhoods is the main expensive operation. SODSS detect outlier by removing cluster objects with stochastically researching. All neighbors in dense neighborhood would not calculate their neighborhood again, so the region query of SODSS must be less than LOF's. Accordingly, SODSS have better efficiency than LOF.

\section{Experimental Evaluation}

The criteria evaluating outlier detection approaches can be divided into two parts: efficiency and effectiveness. Good efficiency means the technique should be applicable not only to small databases of just a few thousand objects, but also to larger databases with more than hundred thousand of objects. As for effectiveness, a good approach should have ability to divide exactly outliers from clusters. We have done many experiments to examine the efficiency and effectiveness, but here limiting to extension we only presented two. In first, we use synthetic data to explain effectiveness of our approach. Secondly, we use large database to verify the efficiency. Experiments showed that our ideas can be used to successfully identify significant local outliers and performance outperforms the other density-based approaches. All experiments were run on a $2.2 \mathrm{GHz}$ PC with $256 \mathrm{M}$ memory. 


\subsection{Effectiveness}

To compare SODSS with GDBSCAN [6] and LOF [7] in terms of effectiveness, we use the synthetic sample databases which are depicted in Fig. 3. In these datasets, the non-spatial property for the points is depicted by different symbol, rings and solid points. Experiment focus on solid objects, and set q.attrs = solid. Fig. 4 shows the outliers and clusters identified by SODSS. The radius set to 2.1 in SODSS, MinPts set to 3. SODSS and GDBSCAN can identify outliers correctly, because they consider non-spatial attribute. As shown in Fig. 5, $L O F$ does not find the outliers because it ignores non-spatial attributes and considers all objects are cluster.

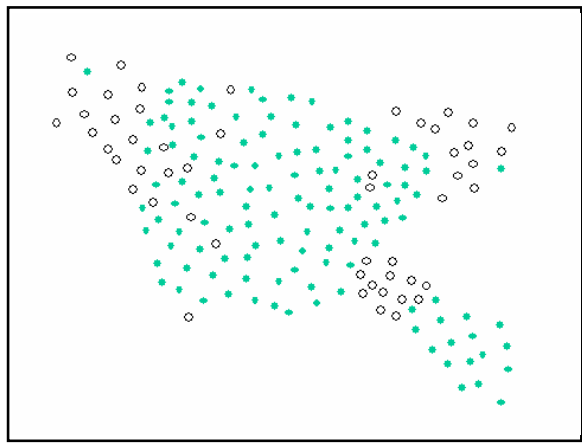

Fig. 3. Synthetic sample databases

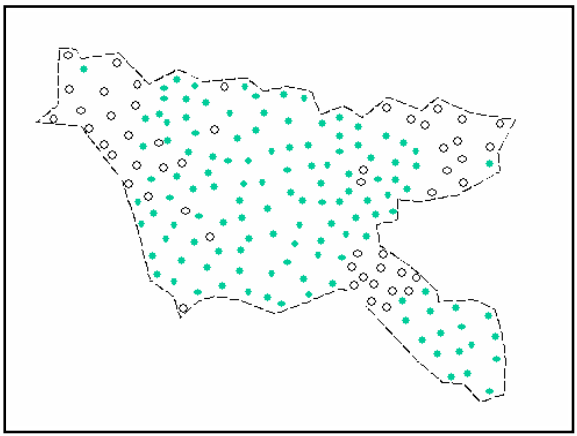

Fig. 5. $L O F$ can't identify outliers

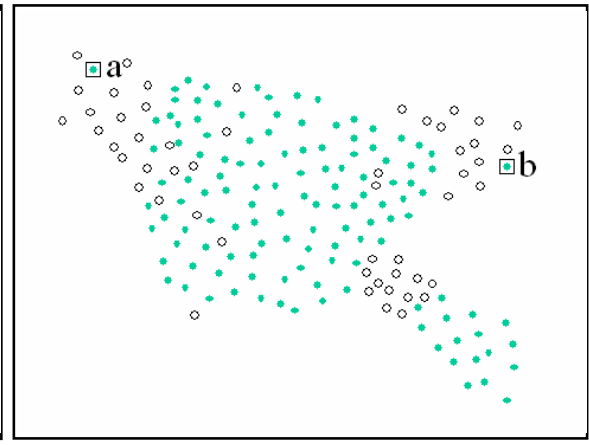

Fig. 4. Outlier $a$ and $b$ identified by SODSS or SDBDCAN

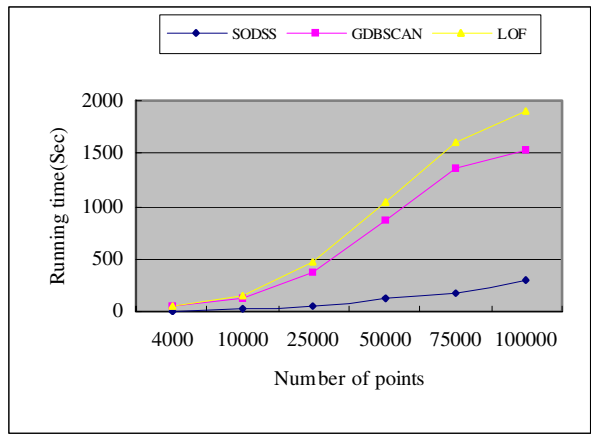

Fig. 6. Time efficiency comparisons between GDBSCAN, LOF and SODSS

\subsection{Efficiency}

For comparison computational efficiency of SODSS and GDBSCAN and $L O F$, we used synthetic datasets that are consisted of points from 4000 to 100,000. The Eps is 5, and MinPts is 10, when SODSS query the neighborhood. They are the same when 
GDBSCAN run. We set MinPts $=30$ and $L O F>1.5$. Fig. 6. shows the running time for $S O D S S$ increases with the size of the datasets in an almost linear fashion, and the performance is obviously better than the other two.

\section{Conclusion}

In this paper, we formulated the problem of one-type spatial outlier detection and presented effective and efficient SODSS algorithms for spatial outlier mining. This algorithm does not calculate neighborhood of very objects but stochastically research. It discards much region query of cluster, and gained good efficiency.

Acknowledgements. This research supported by the National Nature Science Foundation of China (No. 49971063), the National Nature Science Foundation of Jiangsu Province (BK2001045), the High-Tech Research of Jiangsu Province (BG2004005) and the National High-Tech Research and Development Plan of China (No. 2001AA634010-05).

\section{References}

1. D. Hawkins. Identification of Outliers. Chapman and Hall, London, 1980

2. H. Dai, R. Srikant, and C. Zhang. OBE: Outlier by Example. In: Proceedings of PAKDD 2004, Sydney, Australia, May 26-28, 2004, LNAI 3056, pages: 222-234, 2004

3. S. Shekhar, C.T. Lu, and P. Zhang. A unified approach to detecting spatial outliers. GeoInformatica, 7(2): 139-166, 2003

4. C.T. Lu, D. Chen, and Y. Kou. Algorithms for spatial outlier detection. In Proceedings of the 3rd IEEE International Conference on Data Mining (ICDM 2003), December 19-22, 2003, Melbourne, Florida, USA, pages: 597-600. IEEE Computer Society, 2003

5. M. Ester, H.P. Kriegel, J. Sander, and X. Xu. A density-based algorithm for discovering clusters in large spatial databases. In: Proceedings of KDD'96, Portland OR, USA, pages: 226-231, 1996

6. J. Sander, M. Ester, H. Kriegel, and X. Xu. Density-based Clustering in Spatial Databases: the algorithm GDBSCAN and its applications. Data Mining and Knowledge Discovery, val. 2, no. 2, pages: $169-194,1998$

7. M.M. Breunig, H.P.Kriegel, R.T.Ng, and J. Sander. LOF: Identifying density-based local outliers. In: Proceedings of SIGMOD'00, Dallas, Texas, pages: 427-438, 2000

8. N. Beckmann, H.P. Kriegel, R. Schneider, and B. Seeger. The R*-Tree: An Efficient and Robust Access Method for Points and Rectangles. SIGMOD Record, vol. 19, no. 2, pages: 322-331, 1990

9. N. Katayama and S. Satoh. The SR-tree: An Index Structure for High-Dimensional Nearest Neighbor Queries. SIGMOD Record, vol. 26, no. 2, pages: 369-380, 1997 